\title{
Polyamine Plays Key Role in Different Osmotic Stress Responses of Wheat-Rye 1BL/1RS Translocation Lines
}

\author{
K.J. DenG ${ }^{1 *}$, J.P. ZHOU ${ }^{1 *}$, X.H. Wu' ${ }^{2}$, G. Sun ${ }^{1}$, T. WANG ${ }^{1}$, A.T. TANG ${ }^{1}$, \\ X.L. ZHENG ${ }^{1}$ and Y. ZHANG ${ }^{1 * *}$ \\ ${ }^{1}$ School of Life Sciences and Technology, University of Electronic Science and Technology of China, \\ Chendu 610054, China \\ ${ }^{2}$ Shandong Entry-Exit Inspection and Quarantine Bureau, Qingdao 266002, China
}

(Received 28 November 2015; Accepted 7 March 2016;

Communicated by M. Molnár-Láng)

\begin{abstract}
It is well demonstrated that wheat-rye $1 \mathrm{BL} / 1 \mathrm{RS}$ translocated chromosome leads to some valuable novel traits such as disease resistance, high yield and functional stay-green after anthesis. To understand the physiological mechanism of 1BL/1RS translocation responsible for osmotic stress, two wheat cultivars, $\mathrm{CN} 12$ and CN17, carrying the translocated chromosome and MY11 without the translocated chromosome were employed in the study. During 5-day osmotic stress, fresh weight inhibition, chlorophyll content, soluble protein content, MDA concentration, antioxidant enzymes activity and free polyamines content were examined. $\mathrm{CN} 12$ and $\mathrm{CN} 17$, especially cultivar CN17, registered greater biomass and minor oxidative damage compared with their wheat parent. Meanwhile, the concentration of Spd and Spm in CN17 was significantly higher than the others. In addition, we found a positive correlation of fresh weight inhibition (FWI) and Put concentration, and a negative one with the parameters $(\mathrm{Spd}+\mathrm{Spm})$ : Put ratio, indicating the importance of higher polyamine (Spd and $\mathrm{Spm}$ ) accumulation on the adaptation to osmotic stress. Therefore, we proposed that the accumulation of higher polyamines (Spd and Spm) should play an important role on the adaptation of 1BL/1RS translocation lines to osmotic stress and might be important factors for the origin of novel traits introduced by $1 \mathrm{BL} / 1 \mathrm{RS}$.
\end{abstract}

Keywords: wheat, 1BL/1RS translocation lines, osmotic stress, polyamine, physiological parameters

\section{Introduction}

Adverse environmental conditions, including abiotic stress, such as chilling, salt, drought and heavy metals stress, result in significant yield losses in crops (Soltis and Soltis 2009). Introduction of superior alien germplasms is an efficient way to improve crop breeding by generation of novel adaptive traits, providing valuable sources for yield improvement and environment adaptation (Friebe et al. 1996; Ren et al. 2011). Wheat-rye 1BL/1RS translocations have contributed substantially to the world wheat production because of the

\footnotetext{
*These authors contributed equally.

**Corresponding author; E-mail: zhangyong916@uestc.edu.cn
} 
presence of several disease resistance genes and a yield enhancement factor on the rye (Secale cereale L.) chromosome. Nevertheless, the underlying mechanism for the origin of adaptive traits under adverse stress in the derived progenies containing alien chromatin remains to be elucidated. To improve crop breeding under stress, it is necessary to investigate the molecular and biochemical mechanisms by which the derived progenies tolerate environmental stresses.

Plants, when subjected to adverse environmental conditions, undergo alterations in their growth, metabolism, and production. Abiotic stress inhibits plant growth and generates reactive oxygen species (ROS), resulting in damages, such as DNA damage, amino acids oxidation and peroxidation of membrane lipids (Asada 1999; Davies 2003). To avoid such damages and protect the cells, plants have developed ROS-detoxification mechanisms including low molecular weight antioxidants (e.g., phenols, ascorbic acid, glutation) and antioxidant enzymes, such as superoxide dismutase (SOD), catalase (CAT), and peroxidases (POD). Decreased CAT activities under water stress have been observed in sunflower and wheat (Zhang and Kirkham 1994). Sun et al. (2010) studied the behavior of antioxidant enzymes under heavy metal contaminant in radish, observing increased SOD, CAT, and POD activities and isozymes induction. Sánchez-Rodríguez et al. (2010) found also that the activity of CAT in the tomato would have had an important role in preventing the formation of ROS, and therefore achieving better water-deficit tolerance.

Among abiotic stresses, osmotic stress is the most severe environmental factor in agriculture, caused by drought, high salinity and extreme temperatures. One of the most common strategies of plants for avoiding osmotic stress is the production and accumulation of osmolytes for osmotic adjustment (Bohnert et al. 1995). During osmotic stress, plant cells accumulate compatible solutes for the physiological response to prevent water loss and re-establish cell ion homeostasis. The solutes that accumulate during osmotic adjustment include low molecular-weight osmolytes such as proline and polyamines (Tamura et al. 2003; Sánchez-Rodríguez et al. 2010). Polyamines are aliphatic nitrogen compounds positively charged at physiological $\mathrm{pH}$. This polycationic property allows PAs to interact with negatively charged macromolecules as DNA and RNA, proteins and phospholipids and in this way they are involved in the regulation of physical and chemical properties of membranes, nucleic acids structure and functions and modulation of enzyme activities (Galston and Sawhney 1990; Groppa and Benavides 2008). Numerous studies in different plant species have shown that polyamine levels are modulated in response to several abiotic stresses, most remarkably on salt and drought stress (Kusano et al. 2008; Alcazar et al. 2010).

The 1BL/1RS translocation lines, CN12 and CN17 studied in this research had been demonstrated to carry many valuable agronomic traits such as functional stay-green after anthesis and high disease resistance (Tang et al. 2009; Ren et al. 2009, 2011). These translocations had been previously characterized using various cellular and molecular analyses (Luo et al. 2006, 2013; Zhang et al. 2008). However, more studies are needed to better understand the mechanism underlying the origin of these novel traits and to obtain information about the involvement of $1 \mathrm{BL} / 1 \mathrm{RS}$ translocation in the adaptation of plants to 
stress conditions. In the present study, the effect of $1 \mathrm{BL} / 1 \mathrm{RS}$ on physiological parameters, especially polyamine accumulation, were compared in CN12 and CN17 with their wheat parent, MY11 at the seedling stage subjected to osmotic stress.

\section{Materials and Methods}

\section{Plant material}

Two wheat-rye 1BL/1RS translocation lines, CN12 and CN17, were derived from the cross and backcross between Triticum aestivum cv. MY11 and Secale cereale cv. Petkus. CN12 and CN17, which are the predominant cultivars grown in the southwest of China and were released in 2002 and 2003. All samples used in this study were maintained in the laboratory of Prof. Ren at Sichuan Agriculture University, China, and had been previously characterized using various cellular and molecular analyses (Tang et al. 2009).

\section{Growth conditions and fresh weight inhibition experiment}

Plant seedlings of growth conditions was carried out according to Kovács et al. (2010). Osmotic stress was treated with 20\% PEG (w : v) lasted for 5 days, under the cultivation conditions described above. PEG was added to the nutrient solutions, which was changed each day during the whole experiment. Seedling samples were collected for biochemical analysis at $0,0.5,1,1.5,2,3,4$ and 5 days after PEG treatment, and the experiments were repeated three times.

Seedling fresh weight $(\mathrm{FW})$ inhibition rate was calculated according to the following equation:

$$
r=\left(\mathrm{FW}_{\text {control }}-\mathrm{FW}_{\text {treatment }}\right): \mathrm{FW}_{\text {control }}
$$

In the above equation, $r$ is the fresh weight inhibition rate where $r \in[0,1]$. In addition, $\mathrm{FW}_{\text {control }}$ and $\mathrm{FW}_{\text {treatment }}$ are the fresh weight of the control and PEG-treated samples, respectively.

\section{Determination of physiological parameters and antioxidant enzymes activity}

The fresh seedlings from each material were homogenized in a pestle and mortar with $0.05 \mathrm{M}$ sodium phosphate buffer $(\mathrm{pH} 7.8)$ at all time-points assayed during osmotic treatment. The homogenate was centrifuged at $3,000 \times \mathrm{g}$ for 15 min under $4{ }^{\circ} \mathrm{C}$ and the supernatant was used for measuring the contents of chlorophyll, soluble protein and MDA as described by Lichtenthaler (1987), Bradford (1976), and Hodges et al. (1999), respectively. The activity of SOD, POD and CAT were determined following the method used by Giannopolitis and Ries (1977); Sun et al. (2010) and Zhang et al. (2005), respectively. 


\section{Analysis of polyamines}

The procedure of polyamine extraction and derivatization was performed according to the protocol of Flores and Galston (1982). Chromatographic separation was performed on a YMC-Pack ODS-A C18 column using water-acetonitrile gradient elution, at a flow rate of $0.7 \mathrm{ml} / \mathrm{min}$ and $\mathrm{UV}$ detection at $230 \mathrm{~nm}$.

\section{Statistical analysis}

Samples from three independent experiments were analyzed using the statistical computer package of Microsoft Excel 2007. The data were analyzed by one-way ANOVA using SPSS 11.5 (SPSS Inc.) and differences between the means were compared by Fisher's least-significant difference test (LSD).

\section{Results}

\section{Biomass and physiological parameters}

Plant growth was determined as the fresh weight inhibition rate compared with the control groups. At the beginning of osmotic stress $(0.5 \mathrm{~d})$, strong influences were found in MY11 and CN12, as presented an inhibition of $10 \%$ and $6 \%$ with respect to control plants in fresh weight. On the contrary, CN17 showed no significant differences with respect to the well-watered treatment in this growth parameter (Fig. S1*). At the end of five-day osmotic stress, the FWI of MY11 was significantly higher than CN12 and CN17. These results indicated different patterns of FWI alterations in $\mathrm{CN} 12$ and $\mathrm{CN} 17$, in which the FWI of CN12 remained around 15\% after one day of PEG treatment with no obvious fluctuations, while the FWI of CN17 was not obtained at the beginning of treatment $(0.5 \mathrm{~d})$ and then increased as the time of treatment (Fig. S1).

As shown in Fig. 1a, osmotic treatment had no obvious influence on chlorophyll content in seedlings of CN12, CN17 and MY11, except for a significant decrease in the last day of treatment in MY11. Meanwhile, chlorophyll content in CN12 and CN17 was obviously higher than MY11 in the last two days of treatment. At the end of five-day treatment, no significant visible seedling symptoms were observed in CN12 and CN17, while seedlings of MY11 showed obvious yellowing.

In contrast, the soluble protein content of CN12, CN17 and MY11 decreased significantly when the plants were exposed to $20 \%$ PEG treatment (Fig. 1b). Further analysis revealed that the soluble protein content of MY11 and CN12 remained at the lower level during the osmotic treatment, while in $\mathrm{CN} 17$, the content restored to the original level after 2 days' treatment.

The MDA concentration was measured in seedlings as an indicator of oxidative stress in plants. As shown in Fig. 2c, the content of MDA in MY11 increased significantly since

\footnotetext{
*Further details about the Electronic Supplementary Material (ESM) can be found at the end of the article.
} 

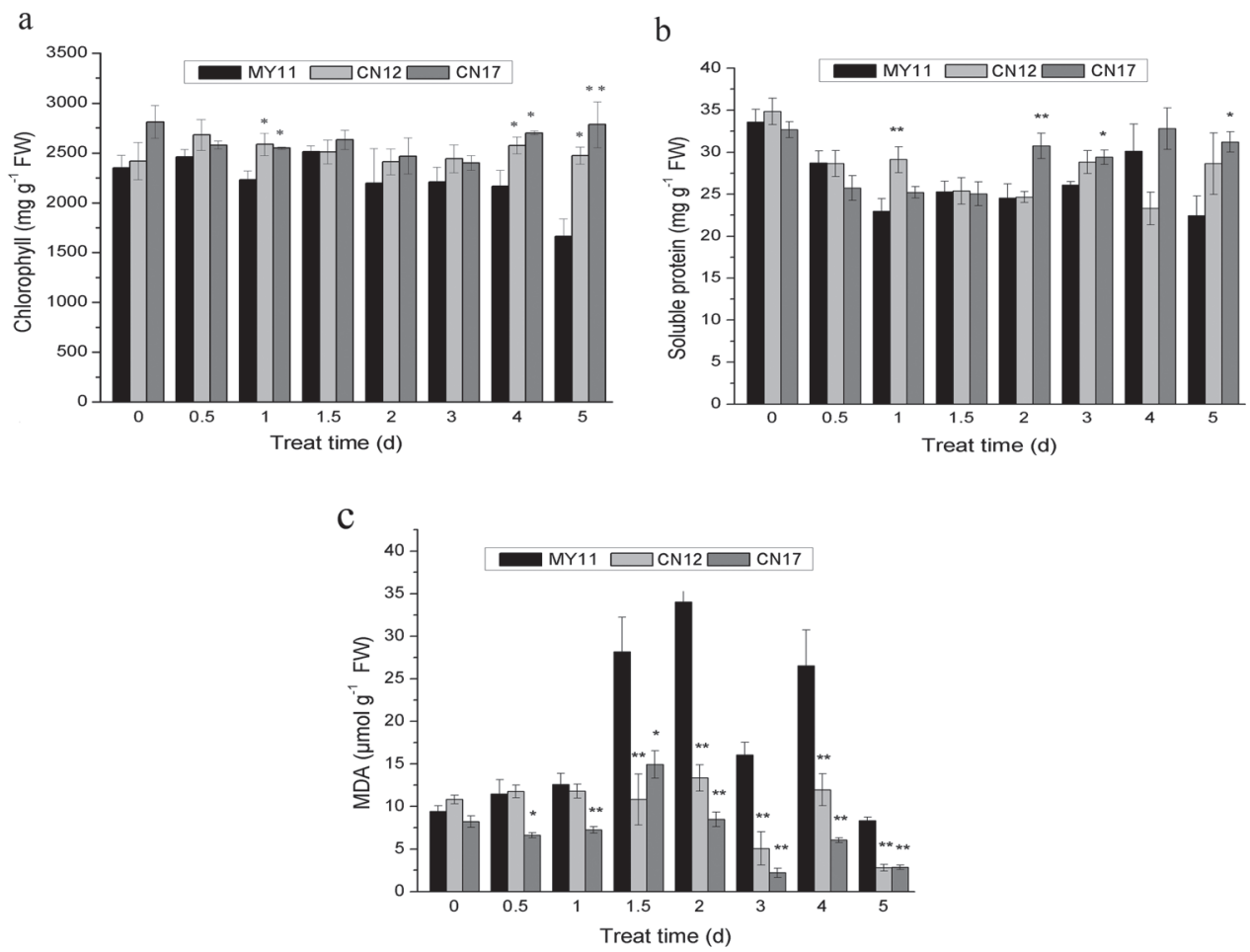

Figure 1. Concentration of (a) chlorophyll, (b) soluble protein and (c) MDA content during 20\% PEG treatment in seedlings of MY11, CN12 and CN17. Columns are mean \pm S.E. $(n=5)$ and differences between means were compared by Fisher's least-significant difference test (LSD). *Significant difference with wheat parent MY11 $(*: P<0.05 ; * *: P<0.01)$

1.5 days' osmotic treatment, which was significantly higher than CN12 and CN17. In contrast, osmotic stress did not induce MDA accumulation in CN12 and CN17.

Totally, the fresh weight inhibition rate and the concentration of chlorophyll, soluble protein and MDA suggested a better growth condition in translocation lines, especially in CN17, than their wheat parent under 20\% PEG-induced osmotic stress.

\section{Activities of antioxidant enzymes}

As shown in Fig. 2a, SOD activities were significantly higher in MY11 than in CN12 and CN17 during the first two days of osmotic treatment. There was an outburst of SOD activities at 3 days after osmotic treatment and remained at this high level in CN12, CN17 and MY11. POD activities were significantly higher in MY11 than in CN12 and CN17, except an obvious decrease in 3 days' treatment. Meanwhile, the variation trend of POD activities was similar in CN12, CN17 and MY11 (Fig. 2b). Osmotic treatment reduced the CAT activities in MY11 at most time point assayed while had no significant influence in 

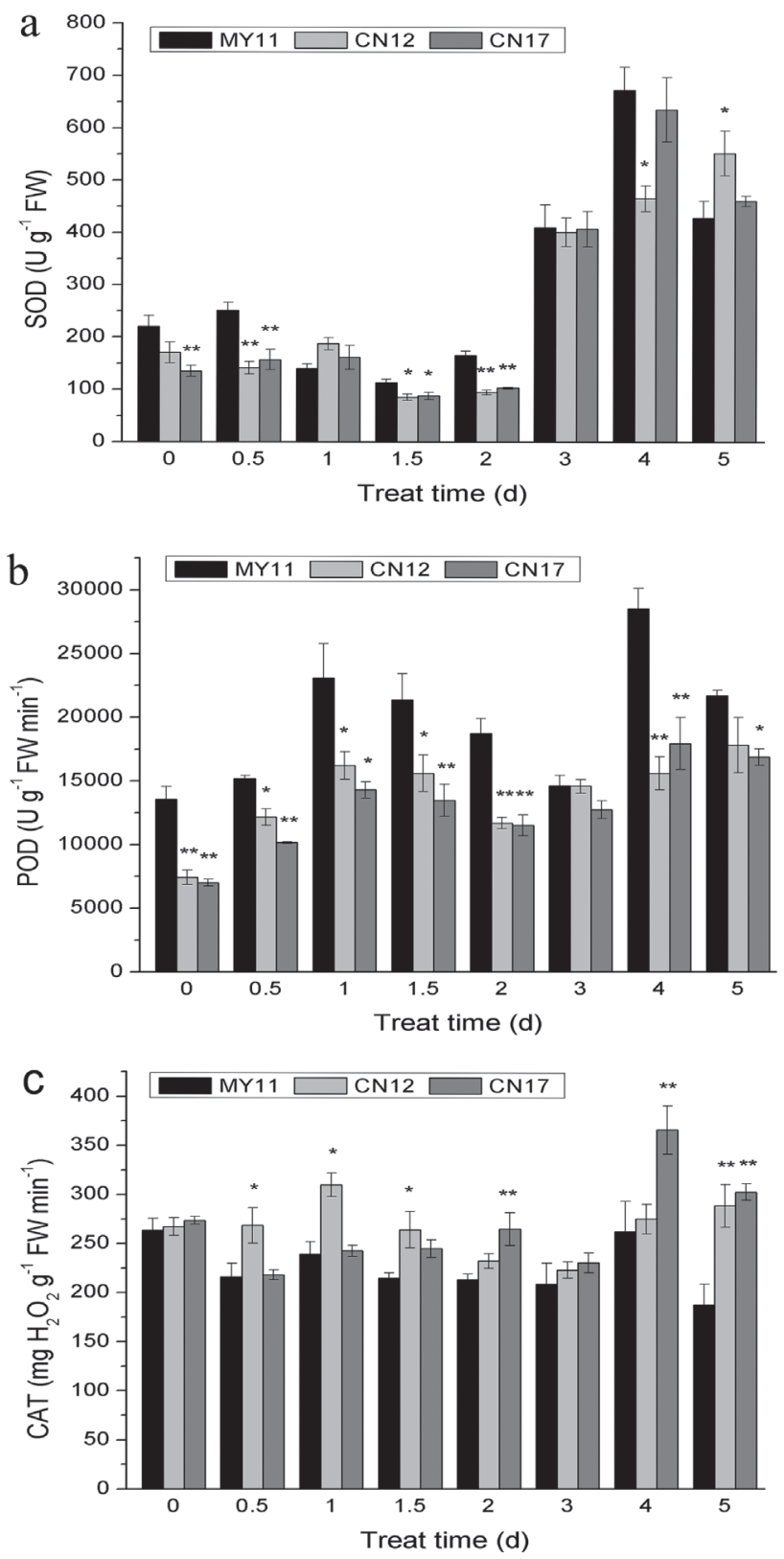

Figure 2. Activities of antioxidant enzymes (a) SOD, (b) POD, (c) CAT during 20\% PEG treatment in seedlings of MY11, CN12 and CN17. Columns are mean \pm S.E. $(n=5)$ and differences between means were compared by Fisher's least-significant difference test (LSD). *Significant difference with wheat parent MY11 $(*: P<0.05 ; * *: P<0.01)$ 

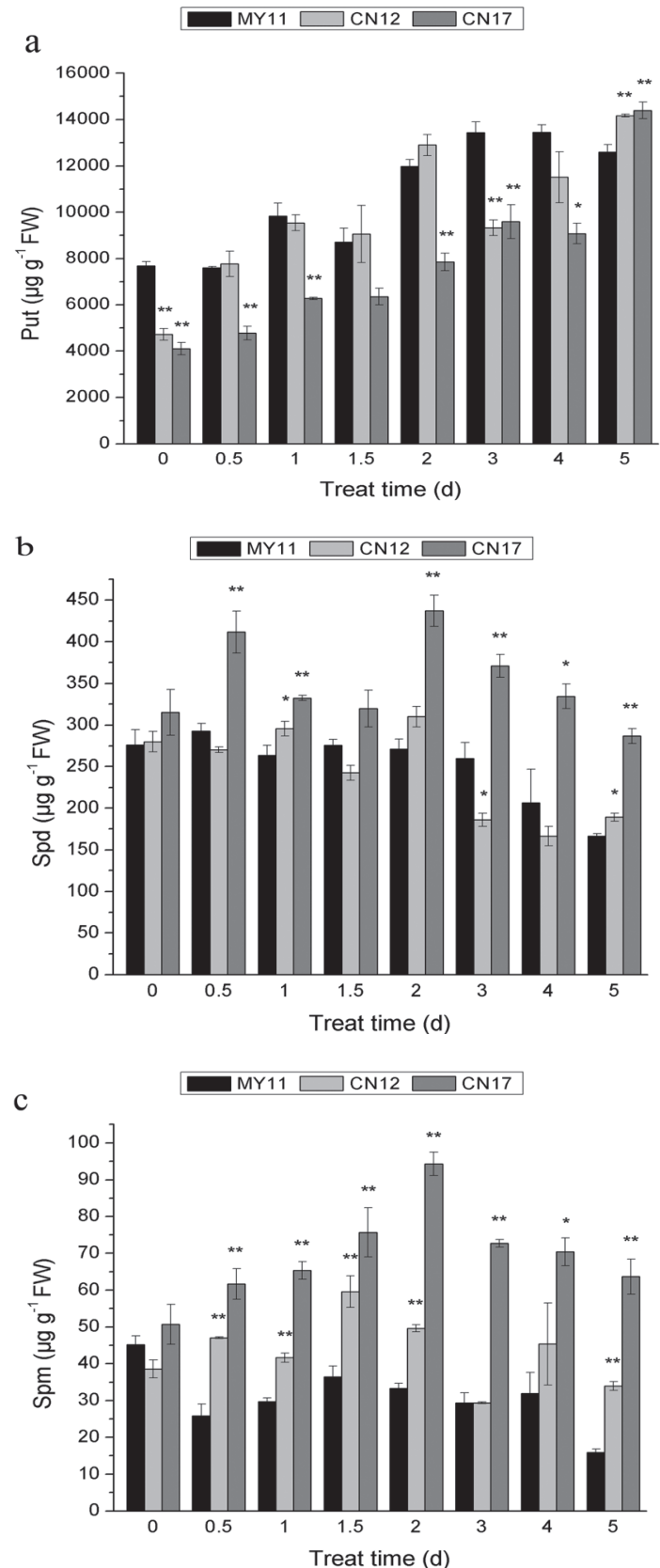

Figure 3. Concentration of three free polyamines (a) Putrescine (Put), (b) Spermidine (Spd), (c) Spermine (Spm) during 20\% PEG treatment in seedlings of MY11, CN12 and CN17. Columns are mean \pm S.E. $(n=5)$ and differences between means were compared by Fisher's least-significant difference test (LSD). *Significant difference with wheat parent MY11 (*: $P<0.05 ; * *: P<0.01)$ 
CN12 (Fig. 2c). Data analysis showed that the CAT activities in CN12 were higher than MY11 in the first two days of treatment, while the CAT activities in CN17 were higher than MY11 in later period of treatment.

\section{Polyamines content}

The concentration of three free polyamines, Put, Spd and Spm, were detected in CN12, CN17 and MY11 with 20\% PEG. The concentration of Put increased in CN12, CN17 and MY11 when the plants were exposed to osmotic stress (Fig. 3a). Put content in MY11 was significantly higher than $\mathrm{CN} 12$ and $\mathrm{CN} 17$ during the treatment, except the last day of treatment. Especially, Put content in CN17 increased smoothly and slowly. The concentration of Spd decreased significantly in MY11 and CN12 in later period of treatment (since 3d), while osmotic stress did not decrease the Spd content in CN17 but induced an increase at $12 \mathrm{~h}, 2 \mathrm{~d}$ and $3 \mathrm{~d}$ of treatment (Fig. 3b). The concentration of Spm decreased significantly in MY11 at the beginning of treatment and maintained low levels. Meanwhile, osmotic treatment had no significant influence on Spm content in CN12 but raised the Spm concentration in CN17 (Fig. 3c). Data analysis showed that the concentration of Spd and Spm in CN17 was significantly higher than the others.

Further analysis of the $(\mathrm{Spd}+\mathrm{Spm})$ : Put ratio revealed different variation patterns of polyamines during 20\% PEG treatment in CN12, CN17 and MY11 (Fig. S2). Before the osmotic treatment, the $(\mathrm{Spd}+\mathrm{Spm})$ : Put ratio in CN12 and CN17 was significantly higher than MY11, in which the ratio in CN17 was twice the ratio in MY11. At the beginning of treatment (12 h), no significant change was obtained in MY11, while the ratio decreased in $\mathrm{CN} 12$ and increased in $\mathrm{CN} 17$. Later, the $(\mathrm{Spd}+\mathrm{Spm})$ : Put ratio decreased slowly in both CN12, CN17 and MY11, and the ratio of CN17 was almost twice the ratio of MY11 and CN12 during the whole treatment.

\section{Correlation analysis}

To determine the relationship between the different parameters indicative of oxidative stress and plant growth parameter fresh weight inhibition (FWI) rate under osmotic stress, we studied the correlation coefficients. We found a positive correlation with Put concentration, and a negative one with the parameters $(\mathrm{Spd}+\mathrm{Spm})$ : Put ratio (Fig. S3).

\section{Discussion}

Introduction of foreign chromatin is associated with the origin of novel traits and confers increased vigor compared with the parent lines especially under environmental stresses (Ren et al., 2011). In this study, we investigated two 1RS/1BL wheat-rye translocation sister lines (CN12 and CN17) and their parent (MY11) under the osmotic stress. In our experiment, both FWI and chlorophyll concentration indicated better growth situation in two translocation lines compared with their parent in later phase of osmotic-stress treatment, especially $\mathrm{CN} 17$, in which the concentration of chlorophyll and soluble protein 
restored to the original level before stress (Fig. 1a, b). Furthermore, the MDA concentration was measured as an indicator of oxidative stress in plants. It was observed that water deficit raised the quantity of MDA in wheat plants (Esfandiari et al. 2007). Meanwhile, low concentrations in MDA have been associated with water-stress tolerance in wheat (Sairam et al. 2000). In our experiment, the same effect that MDA was not accumulation in CN12 and CN17, while the significant increase in the content of MDA in MY11 after 1.5 days' osmotic treatment has been observed (Fig. 1c). Our data suggested a low level of peroxidation (denoted by the MDA concentration) and regular concentrations of physiological parameters (chlorophyll and soluble protein) in $\mathrm{CN} 12$ and $\mathrm{CN} 17$, which indicated osmotic-stress tolerance in these two translocations.

Abiotic stress inhibits plant growth and generates reactive oxygen species (ROS). To control the level of ROS and protect the cells upon exposure to osmotic stress, plants usually show increased SOD, CAT, and POD activities. High activity of these enzymes was demonstrated to conferred tolerance to water deficit (Dhindsa and Matowe 1981; Dhanda et al. 2004). However, our results suggested lower SOD and POD activities in CN12 and CN17 at most time point assayed compared with MY11 (Fig. 2a, b). The CAT activities in $\mathrm{CN} 12$ and CN17 were higher than those in MY11 at some time point assayed, while the change patterns in $\mathrm{CN} 12$ and $\mathrm{CN} 17$ were different either (Fig. 2c). The reasons for this phenomenon might be that CN12, CN17 and MY11 had different osmotic stress threshold and therefore they had different physiological adaptive mechanisms to regulate their redox status (Shao et al. 2007). For example, the content of MDA indicated that the oxidative stress in CN12 and CN17 were much milder than that in MY11, and therefore triggered different ROS-detoxification mechanisms in the plants.

Previous data showed that the effects of cold, osmotic stress and abscisic acid (ABA) on polyamine accumulation were observed in the different wheat cultivars and that some of these changes are suggested to correlate with the level of stress tolerance (Kovács et al. 2010). Meanwhile, there has been a growing interest in the study of the protective role of higher PAs (Spd and Spm) against environmental stresses (Capell et al. 2004). In our study, Put content in MY11 was significantly higher than CN12 and CN17 during the treatment, while osmotic stress did decrease the content of higher Pas, Spd and Spm in MY11 (Fig. 3). On the contrary, the concentration of Spd and Spm in CN17 increased under osmotic stress and was significantly higher than MY11 (Fig. 3). Interesting, further analysis revealed that the $(\mathrm{Spd}+\mathrm{Spm})$ : Put ratio in $\mathrm{CN} 12$ and $\mathrm{CN} 17$ was significantly higher than that in MY11 before the osmotic treatment, in which the ratio in CN17 was twice the ratio in MY11 (Fig. S2). Moreover, the ratio of CN17 was almost twice the ratio of MY11 and CN12 during the whole treatment. We found that the changes of (Spd+ $\mathrm{Spm})$ : Put ratio in CN12 and CN17 was detected earlier than that in MY11, which means a much quicker response to the stress. Totally, our data showed that CN12 and CN17 presented a relatively higher level of higher Pas (Spd and Spm) during the osmotic stress treatment, whereas MY11 maintained only a high level of Put. Further analysis of the correlation between FWI and other parameters indicated the important role of higher polyamine (Spd and Spm) accumulation on the adaptation of CN12 and CN17 to osmotic stress (Fig. S3). 
In conclusion, we found that the $1 \mathrm{RS} / 1 \mathrm{BL}$ translocation lines, which showed many novel agriculture traits, also presented growing vigor at the seedling stage in the response to PEG-induced osmotic stress. The translocations, especially CN17, presented a better biomass together with minor oxidative damage associated with a stronger response of osmotic adjustment by polyamines. Moreover, we concluded that the accumulation of higher polyamines (Spd and Spm) should play an important role in the adaptation of these translocations to osmotic stress and might be important factors for the origin of novel traits. Therefore, our studies about the involvement of polyamines are necessary to understand the mechanism underlying the origin of novel adaptive traits which was induced by foreign chromatin when subjected to environmental stresses.

\section{Acknowledgements}

This work was supported by the National Natural Science Foundation of China (Nos. 31271420, 30900779 and 31371682), the National Transgenic Major Project (2014ZX0801003B-002) and the Fundamental Research Funds for the Central Universities (ZYGX2013J099).

\section{References}

Alcazar, R., Altabella, T., Marco, F., Bortolotti, C., Reymond, M., Koncz, C., Carrasco, P., Tiburcio, A.F. 2010. Polyamines: Molecules with regulatory functions in plant abiotic stress tolerance. Planta 231:1237-1249.

Asada, K. 1999. The water-water cycle in chloroplasts: Scavenging of active oxygens and dissipation of excess photons. Annu. Rev. Plant Physiol. Plant Mol. Biol. 50:601-639.

Bohnert, H.J., Nelson, D.E., Jensen, R.G. 1995. Adaptations to environmental stresses. Plant Cell 7:1099-1111.

Bradford, M.M. 1976. A rapid and sensitive method for the quantitation of microgram quantities of protein utilizing the principle of protein-dye binding. Anal. Biochem. 72:248-254.

Capell, T., Bassie, L., Christou, P. 2004. Modulation of the polyamine biosynthetic pathway in transgenic rice confers tolerance to drought stress. Proc. Natl Acad. Sci. USA 101:9909-9914.

Davies, M.J. 2003. Singlet oxygen-mediated damage to proteins and its consequences. Biochem. Biophys. Res. Commun. 305:761-770.

Dhanda, S.S., Sethi, G.S., Behl, R.K. 2004. Indices of drought tolerance in wheat genotypes at early stages of plant growth. J. of Agron. and Crop Sci. 190:6-12.

Dhindsa, R.S., Matowe, W. 1981. Drought tolerance in two mosses: correlated with enzymatic defence against lipid peroxidation. J. Exp. Bot. 32:79-91.

Esfandiari, E.O., Shakiba, M.R., Mahboob, S.A., Alyari, H., Toorchi, M. 2007. Water stress, antioxidant enzyme activity and lipid peroxidation in wheat seedling. J. of Food Agric. and Environ. 5:149-153.

Flores, H.E., Galston, A.W. 1982. Analysis of polyamines in higher plants by high performance liquid chromatography. Plant Physiol. 69:701-706.

Friebe, B., Jiang, J., Raupp, W., McIntosh, R., Gill, B. 1996. Characterization of wheat-alien translocations conferring resistance to diseases and pests: current status. Euphytica 91:59-87.

Galston, A.W., Sawhney, R.K. 1990. Polyamines in plant physiology. Plant Physiol. 94:406-410.

Giannopolitis, C.N., Ries, S.K. 1977. Superoxide dismutases: II. Purification and quantitative relationship with water-soluble protein in seedlings. Plant Physiol. 59:315-318.

Groppa, M.D., Benavides, M.P. 2008. Polyamines and abiotic stress: recent advances. Amino Acids 34:35-45.

Hodges, D.M., DeLong, J.M., Forney, C.F., Prange, R.K. 1999. Improving the thiobarbituric acid-reactivesubstances assay for estimating lipid peroxidation in plant tissues containing anthocyanin and other interfering compounds. Planta 207:604-611. 
Kovács, Z., Simon-Sarkadi, L., Szűcs, A., Kocsy, G. 2010. Differential effects of cold, osmotic stress and abscisic acid on polyamine accumulation in wheat. Amino Acids 38:623-631.

Kusano, T., Berberich, T., Tateda, C., Takahashi, Y. 2008. Polyamines: essential factors for growth and survival. Planta 228:367-381.

Lichtenthaler, H.K. 1987. Chlorophyll and carotenoids: pigments of photosynthetic biomembranes. Methods Enzymol. 148:350-382.

Luo, P.G., Ren, Z.L., Wu, X.H., Zhang, H.Y., Zhang, H.Q., Feng, J.A. 2006. Structural and biochemical mechanism responsible for the stay-green phenotype in common wheat. Chinese Sci. Bulletin 51:25952603.

Luo, P.G., Deng, K.J., Hu, X.Y., Li, L.Q., Li, X., Chen, J.B., Zhang, H.Y., Tang, Z.X., Zhang, Y., Sun, Q.X., Tan, F.Q., Ren, Z.L. 2013. Chloroplast ultrastructure regeneration with protection of photosystem II is responsible for the functional 'stay-green' trait in wheat. Plant Cell Environ. 36:683-696.

Ren, T.H., Yang, Z.J., Yan, B.J., Zhang, H.Q., Fu, S.L., Ren, Z.L. 2009. Development and characterization of a new 1BL.1RS translocation line with resistance to stripe rust and powdery mildew of wheat. Euphytica 169:207-213.

Ren, T.H., Chen, F., Zhang, H.Q., Yan, B., Ren, Z.L. 2011. Application of 1RS.1BL translocation in the breeding of "Chuangnong" series wheat cultivars. J. Triticeae Crops 31:430-436.

Sairam, R.K., Srivastava, G.C., Saxena, D.C. 2000. Increased antioxidant activity under elevated temperatures: a mechanism of heat stress tolerance in wheat genotypes. Biologia Plantarum 43:245-251.

Sánchez-Rodríguez, E., Rubio-Wilhelmi, M., Cervilla, L.M., Blasco, B., Rios, J.J., Rosales, M.A., Romero, L., Ruiz, J.M. 2010. Genotypic differences in some physiological parameters symptomatic for oxidative stress under moderate drought in tomato plants. Plant Sci. 178:30-40.

Shao, H.B., Chu, L.Y., Wu, G., Zhang, J.H., Lu, Z.H., Hu, Y.C. 2007. Changes of some anti-oxidative physiological indices under soil water deficits among 10 wheat (Triticum aestivum L.) genotypes at tillering stage. Colloids and Surfaces B-Biointerfaces 54:143-149.

Soltis, P.S., Soltis, D.E. 2009. The role of hybridization in plant speciation. Annu. Rev. Plant Biol. 60:561-588. Sun, B.Y., Kan, S.H., Zhang, Y.Z., Deng, S.H., Wu, J., Yuan, H., Qi, H., Yang, G., Li, L., Zhang, X.H., Xiao, H., Wang, Y.J., Peng, H., Li, Y.W. 2010. Certain antioxidant enzymes and lipid peroxidation of radish (Raphanus sativus L.) as early warning biomarkers of soil copper exposure. J. of Hazardous Materials 183:833-838.

Tamura, T., Hara, K., Yamaguchi, Y., Koizumi, N., Sano, H. 2003. Osmotic stress tolerance of transgenic tobacco expressing a gene encoding a membrane-located receptor-like protein from tobacco plants. Plant Physiol. 131:454-462.

Tang, Z.X., Fu, S.L., Ren, Z.L., Zhang, H.Q., Yang, Z.J., Yan, B.J. 2009. Characterization of three wheat cultivars possessing new 1BL.1RS wheat-rye translocations. Plant Breeding 128:524-527.

Zhang, H.Y., Jiang, Y.N., He, Z.Y., Ma, M. 2005. Cadmium accumulation and oxidative burst in garlic (Allium sativum). J. Plant Physiol. 162:977-984.

Zhang, J.X., Kirkham, M.B. 1994. Drought-stress-induced changes in activities of superoxide-dismutase, catalase, and peroxidase in wheat species. Plant Cell Physiol. 35:785-791.

Zhang, Y., Liu, Z.H., Liu, C., Yang, Z.J., Deng, K.J., Peng, J.H., Zhou, J.P., Li, G.R., Tang, Z.X., Ren, Z. L. 2008. Analysis of DNA methylation variation in wheat genetic background after alien chromatin introduction based on methylation-sensitive amplification polymorphism. Chinese Sci. Bulletin 53:58-69. 


\section{Electronic Supplementary Material (ESM)}

Electronic Supplementary Material (ESM) associated with this article can be found at the website of CRC at http://www.akademiai.com/content/120427/

Electronic Supplementary Figure S1. Fresh weight inhibition (FWI) rate of MY11, CN12 and CN17 subjected to osmotic treatments. FW inhibition was calculated according to the following equation $r=\left(\mathrm{FW}_{\text {control }}-\right.$ $\left.\mathrm{FW}_{\text {treatment }}\right): \mathrm{FW}_{\text {control. }}$. Here, $r$ is the $\mathrm{FW}$ inhibition rate and $\mathrm{FW}_{\text {control }}$ and $\mathrm{FW}_{\text {treatment }}$ are the fresh weight of the control and osmotic treatments, respectively. Data are mean \pm S.E. $(n=5)$

Electronic Supplementary Figure S2. The $(\mathrm{Spd}+\mathrm{Spm})$ : Put ratio during the 20\% PEG treatment in seedlings of MY11, CN12 and CN17. Data are mean \pm S.E. $(n=5)$

Electronic Supplementary Figure S3. The relationship of fresh weight inhibition (FWI) with physiological parameters: Put content and the $(\mathrm{Spd}+\mathrm{Spm})$ : Put ratio. (a) The relationship of these parameters in wheat parent MY11; (b) The relationship in CN12; (c) The relationship in CN17 